\title{
Evolution of active region outflows throughout an active region lifetime
}

\author{
L. Zangrilli ${ }^{1}$ and G. Poletto ${ }^{2}$ \\ ${ }^{1}$ Istituto Nazionale di Astrofisica (INAF) - Osservatorio Astrofisico di Torino, via Osservatorio 20, 10025 Pino Torinese, Italy \\ e-mail: luca.zangrilli@inaf.it \\ ${ }^{2}$ Istituto Nazionale di Astrofisica (INAF) - Osservatorio Astrofisico di Arcetri, largo Enrico Fermi 5, 50125 Firenze, Italy \\ Received 2 March 2016 / Accepted 18 July 2016
}

\begin{abstract}
Context. We have shown previously that SOHO/UVCS data allow us to detect active region (AR) outflows at coronal altitudes higher than those reached by other instrumentation. These outflows are thought to be a component of the slow solar wind.

Aims. Our purpose is to study the evolution of the outflows in the intermediate corona from AR 8100, from the time the AR first forms until it dissolves, after several transits at the solar limb.

Methods. Data acquired by SOHO/UVCS at the time of the AR limb transits, at medium latitudes and at altitudes ranging from 1.5 to $2.3 R_{\odot}$, were used to infer the physical properties of the outflows through the AR evolution. To this end, we applied the Doppler dimming technique to UVCS spectra. These spectra include the H I Ly $\alpha$ line and the O VI doublet lines at 1031.9 and $1037.6 \AA$.

Results. Plasma speeds and electron densities of the outflows were inferred over several rotations of the Sun. AR outflows are present in the newly born AR and persist throughout the entire AR life. Moreover, we found two types of outflows at different latitudes, both possibly originating in the same negative polarity area of the AR. We also analyzed the behavior of the Si XII $520 \AA$ A line along the UVCS slit in an attempt to reveal changes in the $\mathrm{Si}$ abundance when different regions are traversed. Although we found some evidence for a Si enrichment in the AR outflows, alternative interpretations are also plausible.

Conclusions. Our results demonstrate that outflows from ARs are detectable in the intermediate corona throughout the whole AR lifetime. This confirms that outflows contribute to the slow wind.
\end{abstract}

Key words. solar wind - Sun: UV radiation - Sun: activity

\section{Introduction}

The recent observations of outflows originating from the edges of active regions (ARs) contributed to identify one of the possible sources of the slow solar wind, whose origin has not yet been unambiguously identified. However, although they have been observed in many circumstances, the characteristics of upflows are not well known, nor is it established whether all reach the interplanetary space, or if there are motions that rise along one leg and descend along the other leg of a loop.

By using interplanetary scintillation analysis, Kojima et al. (1999) found slow wind flows during the minimum of solar activity. These were associated with open magnetic field lines that apparently originated in one of the polarities of an AR. Observations with the TRACE satellite have sporadically shown outflows from ARs (Winebarger et al. 2001). The socalled AR sources have been identified as slow wind contributors from the study of Ulysses and ACE in situ observations (see, e.g., Neugebauer et al. 2002; Liewer et al. 2004). According to the analysis made by Neugebauer et al. (2002), the AR outflows can be comprised of different substreams, originating from multiple sites in the AR, and the distribution of open magnetic field lines can change with time.

More recently, continuous outflows from the edges of ARs have been identified through Hinode/XRT (Sakao et al. 2007) and Hinode/EIS observations (Harra et al. 2008; Doschek et al. 2008). All these observations refer to low coronal levels. Analyses of Hinode/EIS line asymmetries (Tian et al. 2011b), combined with SDO/AIA space-time plots, suggested the occurrence of two velocity components: a background primary component (possibly blueshifted by $\approx 10 \mathrm{~km} \mathrm{~s}^{-1}$ ) and a secondary high speed $\left(\approx 100 \mathrm{~km} \mathrm{~s}^{-1}\right)$ component, the latter only possibly reaching high coronal levels (Tian et al. 2011b). Furthermore, it is not clear whether the flows are continuous or quasi-periodic (De Pontieu \& McIntosh 2010; Tian et al. 2011a).

Several mechanisms have been proposed as drivers of the AR outflows, most of them involving magnetic reconnection (see, e.g., Harra et al. 2008; Marsch et al. 2008; Del Zanna 2008). It has been pointed out by Baker et al. (2009) that the strongest AR outflows originate from areas in the proximity of magnetic field configurations that are known as quasi-separatrix layers (QSLs; see, e.g., Demoulin et al. 1996). These authors argued that the magnetic reconnection process, which operates in QSLs and separates AR loops from open magnetic field lines or larger-scale loops, is a possible mechanism underlying the AR outflows. A different mechanism for outflows, based on AR expansion, has been suggested by Murray et al. (2010).

It can be determined whether the outflows detected in the low corona reach the interplanetary medium by comparing the properties of plasma originating at the periphery of ARs and those of the in situ wind plasma, which are assumed to originate from that source. This approach avoids the problem of measuring plasma flows high up in the corona. The method of comparing the low corona plasma properties with the wind parameters measured in situ has been adopted by Ko et al. (2006), for example, who used SOHO/UVCS data to compare abundances inferred in the corona 
at the AR edges with abundances measured in the wind from ACE/SWICS. Analogously, Liewer et al. (2004) analyzed ACE and Ulysses data and concluded that the $\mathrm{O}^{7+} / \mathrm{O}^{6+}$ ratio (a proxy for the electron temperature of the region where freezing-in occurs) is higher and more variable in the solar wind streams that are thought of as originating from ARs than the ratio detected from coronal hole $(\mathrm{CH})$ sources.

An indirect evidence that low corona outflows extend into inteplanetary space has been proposed by Del Zanna et al. (2011) and Bradshaw et al. (2011), who compared Hinode/EIS observations with the radio noise storms mapped by the Nancay radioheliograph. In the scenario proposed by these authors, the observed noise storms originate in low-energy electron beams propagating into the high corona, where they are accelerated by interchange reconnection between closed and open loops in the low corona.

A multi-instrument approach in the study of AR outflows has been adopted by Vanninathan et al. (2015), who excluded the role of chromospheric jets as possible drivers and instead suggested that the magnetic field diffusion might be responsible for the upflows. Moreover, these authors obtained evidence of two outflow regimes from observing two velocity components that they ascribed to magnetic reconnection and a pressure-driven mechanism.

It is well established that the element abundances with a low first ionization potential (FIP), that is, lower than $10 \mathrm{eV}$, in the slow solar wind are significantly higher than those of the photosphere (see, e.g., von Steiger et al. 2000). The degree of enhancement of a particular element is commonly evaluated by the FIP bias factor, which is the ratio of the abundance of this element relative to that of a high-FIP (i.e., with a first ionization potential higher than $10 \mathrm{eV}$ ) reference element measured in the corona, with the same relative abundance as measured in the photosphere. By comparing the abundances determined by Hinode/EIS and SWICS, Brooks \& Warren (2011) made a direct connection between the plasma flowing out from the edge of an AR with the slow wind detected in situ. These authors found that this AR outflow is characterized by values of the FIP bias of $\sim 3-$ 4 , which distinguishes it from the bias derived from observations of the polar $\mathrm{CH}$. These show values of $\sim 1.0-1.1$, as expected for the fast wind (see also van Driel-Gesztelyi et al. 2012; Wang 2012).

A recent full-Sun study, combining the analysis of coronal plasma composition, Doppler outflow speed mesurements, large-scale coronal magnetic field reconstruction, and in situ measurements of the solar wind at $1 \mathrm{AU}$, has been presented by Brooks et al. (2015). By analyzing Hinode/EIS and ACE/SWEPAM data, they produced a solar wind source map that confirmed the role of ARs as slow wind sources. The authors concluded that ARs appear to be the primary contributors to the slow wind flows.

Plasma flows at the intermediate corona levels, in the range from 1.5 to $2.5 R_{\odot}$, have recently been detected by Zangrilli \& Poletto (2012), who analyzed SOHO/UVCS coronal observations at the time when an AR transits at the solar limb. Applying the Doppler dimming (DD) technique to data that covered the NOAA 8124 AR complex on January 2, 1998, these authors showed that outflows were present in a narrow channel at the edge of a closed loop system, where, according to magnetic potential field extrapolations, bundles of open field lines connected the AR to the interplanetary space.

The temporal evolution of AR upflows has so far been studied in the relatively short time interval of a few days. Démoulin et al. (2013) analyzed upflows from the edge of AR 10978 during its transit over the solar disk. They found that the upflows are characterized by a collimated and stationary component, and that their limb-to-limb evolution is dominated by projection effects onto the line of sight (LOS). Their results are also compatible with an origin of the upflows from reconnection along quasi-separatrix layers. Culhane et al. (2014) followed the evolution of the plasma upflows from ARs over the days of its central meridian passage. The authors found a short timescale variability of the upflows and a strong stationary component and confirmed that AR upflows can contribute to the slow solar wind.

In the present work we address the neglected question of how plasma outflows evolve in the intermediate corona on a longer timescale by analyzing successive limb transits of an AR in a time interval of several months, from the time of its birth to the time of its death. We do not have to cope with projection effects on the solar disk because we only use limb observations and focus on possible changes that occurr in the time intervals from one limb transit to the next. Our purpose is to establish whether the deduced outflow speeds and electron densities are a function of the AR age or if they occur at typical speed and density levels, independent of the AR evolution. We also examine the location from which plasma originates to determine whether it changes with time or persists throughout the AR lifetime. A change in the AR outflow sources pattern might depend on a variation in the AR polarities orientation with time and on the AR magnetic field dispersal with the AR age, the latter perhaps leading to a unipolar field or to several low-lying closed loops with no escaping plasma. The evolution of the outflow properties might also depend on the activity level of the AR, as the occurrence of many flares or coronal mass ejections (CME) might indicate the likelihood of magnetic field lines opening. Finally, we analyze the brightness of the $520 \AA$ second-order line of the Si XII ion, which is detected in the O VI channel spectra, and discuss the origin of its changes along the UVCS slit.

The paper is organized as follows: in Sect. 2 we describe the data, in Sect. 3 we summarize the procedure by which we inferred the physical parameters of the AR and, in particular, those of the outflowing plasma. Section 4 describes the results, which are discussed in Sect. 5, where a summary of the main results and the conclusions are also given.

\section{Data}

\subsection{NOAA 8100 active region}

We here study NOAA AR 8100 , which is an interesting and fairly active region that has been the subject of several papers (see, e.g., Yan et al. 2001; Green et al. 2002; Mandrini et al. 2004), which mainly addressed questions on its topology and the energy and helicity budget. Most of the literature refers to November 8, 1997, as the first limb passage of this region, when it was born as AR 8100 a few days before; however, the region had already been seen at the limb of the Sun on October 26-27 (Solar Geophysical Data n. 644 lists a flare observed at the Meudon Observatory from AR 8100, at S20, E75, on October 27, 1997) and made its last limb transit around February 28,1998 . In the following we refer to this active region as AR 8100, throughout its limb passages, although it was assigned different numbers at successive transits: it is called AR 8112, AR 8124, and AR 8142 in the second to fourth rotations, after which no number was assigned to the AR. The estimated number of flares and CMEs originating from the region is possibly higher than 50. The AR magnetic field exhibits a peculiar evolution as its polarities continued to rotate throughout the AR lifetime, 
changing from an $\approx \mathrm{E} / \mathrm{W}$ to an $\approx \mathrm{N} / \mathrm{S}$ orientation (see Fig. 6 in Mandrini et al. 2004). This behavior is very convenient for our analysis, allowing us to see loops both edge-on and face-on, respectively, thus facilitating our understanding of the 3D structure of the region.

\section{2. $S O H O / U V C S$ data}

SOHO/UVCS (see, e.g., Domingo et al. 1995; Kohl et al. 1995, $1997,2006)$ routinely made synoptic observations of the Sun, acquiring spectra at eight different polar angles (PAs) every 45 degrees, starting from the north pole. At every PA, the UVCS slit is set normal to the radial of the Sun, at a number of heliocentric distances that start at $1.5 R_{\odot}$ and reach $3.5 R_{\odot}$ at the equator, and lower altitudes at the other PAs, where weaker line intensities are expected. Typically we used data taken at PA $=135 \mathrm{deg}$ and $\mathrm{PA}=225 \mathrm{deg}$, corresponding to the latitude of $-45 \mathrm{deg}$ (southern hemisphere), SE and SW quadrant, respectively, for the east and west limb passages. The radial from the Sun center, which defines the PA, intercepts the spectrograph slit in an off-centered position. The resulting unequal distribution of the pixels along the spatial direction, relatively to the slit PA, implies a shorter coverage of the corona at low latitudes in the SE quadrant observations than in the SW. However, at the east limb the AR is marginally within the field of view of the observations taken with the slit at PA $=90 \mathrm{deg}$ (and also at $135 \mathrm{deg}$ ), which are made along the equatorial direction. This circumstance, and the need of a wider coverage of the corona at low latitudes, caused us to use both mid-latitudes and equatorial scans when analyzing the transits at the east solar limb.

SOHO/UVCS acquired data over the ten east and west limb transits of the AR, from the end of October 1997 to the end of February 1998. The passage at the west limb on January 2, 1998, has been analyzed by Zangrilli \& Poletto (2012). We note in passing that since UVCS takes data only at the solar limb, when there is no direct way of knowing the magnetic field at the base of the region, any comparison between the UVCS structure and the magnetic field reconstructions (based on data collected throughout a solar rotation) is subject to some margin of uncertainty (see discussion in Sect. 3.3).

The field of view covered by UVCS $\left(40^{\prime}\right.$ along the slit direction) is shown in Fig. 1, in which the four slit positions and the data binning along the spatial direction are also given. The fixed edge of the UVCS slit, whose projections are represented in the cartoon as green rectangles, was set at 1.5, 1.8, 2.1 and $2.4 R_{\odot}$. In the following, we refer to these slit positions as positions 1, 2, 3 and 4. Data are acquired on two consecutive days, when synoptic data are available (with the only exception of the sixth transit on January 2, 1998, owing to the lack of synoptic data over two consecutive days), and have been summed up over a three-degree wide sun-centered angular sector to increase the signal-to-noise ratio. The synoptic observations typically last only about $45 \mathrm{~min}$ over all four slit positions. This time is often not long enough to guarantee a good statistics, particularly at positions 3 and 4 , and at relatively high latitudes. Hence, we summed data over two consecutive synoptics after ensuring, by inspecting SOHO/LASCO images (Brueckner et al. 1995), that the spatial coronal structures along the slit were stable enough to allow the data combination. Zangrilli \& Poletto (2012) summed the spatial data over a number of pixels that was the same along the slit for all the four positions, spanning a decreasing latitude interval with distance. In the present study, the choice of a constant angular width of the bins at all slit positions allows partially compensating for the decreasing count rate in spectra with

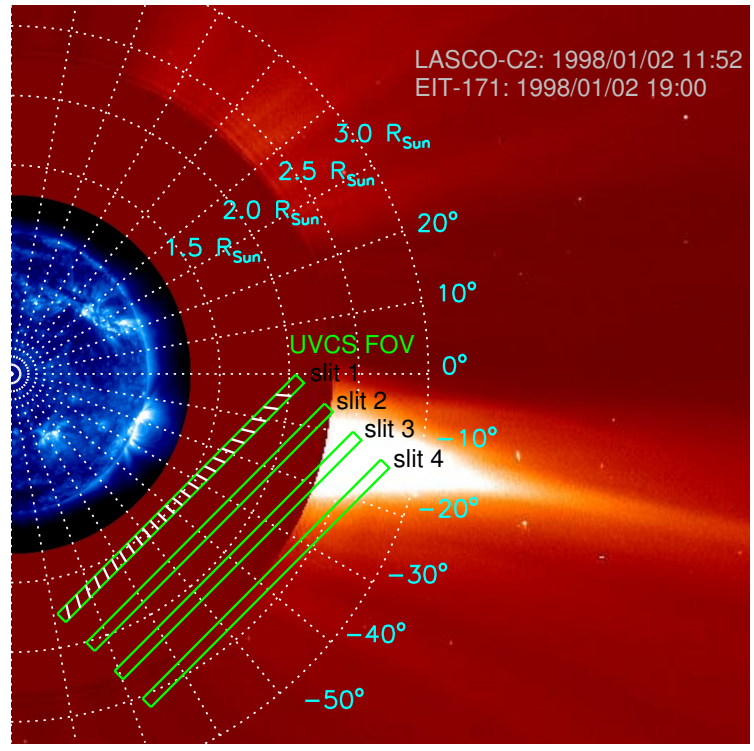

Fig. 1. Cartoon illustrating the configuration of the UVCS O VI channel observations at the west limb: the UVCS field of view is shown in green as the projection onto the plane of the sky of the slit at the four positions. As an example of the data binning along the spatial direction, the slit at $1.5 R_{\odot}$ is divided into the three-degree wide angular sectors within which UVCS data have been summed. The slit positions at the two PAs at the east limb are illustrated in the eastern panels of Fig. 2. As context images, we show an image from EIT-171, taken on January 2, 1998 when AR 8100 was at the west limb at a latitude of about $-20 \mathrm{deg}$, and a LASCO-C2 image, taken on the same day.

heliocentric distance and matching the observations at medium latitudes with those taken along the equatorial direction, which we used here.

UVCS data have been taken in the H I Ly $\alpha$ and O VI channels, which have different characteristics: in the radial direction, the $\mathrm{O}$ VI channel slit width is $0.3 \mathrm{~mm}$, while the H I Ly $\alpha$ channel slit width is $50 \mu \mathrm{m}$, thus covering different intervals of heliocentric distances, corresponding to $83^{\prime \prime}$ and $14^{\prime \prime}$ in the plane of the sky, respectively. Data have originally been acquired with a spatial resolution of $21^{\prime \prime}$ per bin in the plane of the sky, which has then been degraded by averaging data over three-degree wide sun-centered latitude sectors, as we discussed above.

Spectra acquired in the $\mathrm{O}$ VI channel include the doublet $\mathrm{O}$ VI lines at 1031.9 and $1037.6 \AA$, the $\mathrm{H}$ I Ly $\beta$ line at $1025.7 \AA$, and the weaker line of the Si XII doublet at $520.7 \AA$ (in the second order). The spectral resolution of O VI data is $0.594 \AA$ per bin. In the H I Ly $\alpha$ channel the spectral resolution is $0.14 \AA$ per bin. We point out that data are taken with exposure times that increase with the slit heliocentric altitude in an attempt to make counts at the largest distances accumulate long enough to become statistically significant. However, as the distance from the Sun increases along the slit as well (it is higher for pixels at the slit ends), there are cases when the exposure time is not long enough to provide significant data all along the slit, hence these positions were not considered in the study.

Data were analyzed using the UVCS - Data Analysis Software, which takes care of wavelength and radiometric calibration. Stray-light effects have been corrected through the semiempirical relationship given by Cranmer et al. (2010). Line intensities were calculated after fitting the observed line profiles with a Gaussian and then integrating over wavelength. 


\subsection{SOHO/EIT context images}

As context data, we used wavelet-enhanced images from SOHO/EIT (Delaboudinière et al. 1995) to infer the loop configurations in the low corona at the times when the AR crosses the limb of the Sun. EIT images were acquired in the 195 and $171 \AA$ wavebands, and, as far as possible, at the same dates as the SOHO/UVCS data we analyzed. The occurrence of weaker emission regions in EIT images might help us to identify the source regions of outflows, if any. Long-lived upflows have been recognized in Hinode/EIS analyses to correspond to low-density and low-electron temperature regions (e.g., Doschek et al. 2008; Del Zanna 2008; Hara et al. 2008; Démoulin et al. 2013). In particular, Doschek et al. (2008) found evidence that low-intensity regions in ARs are significantly Doppler-shifted and are associated with open magnetic field lines that extend into the heliosphere; the estimated electron densities and temperatures in the outflows are considered rather low for an active region. We therefore expect to find the low-corona counterparts of the outflows we detect in the intermediate corona as weaker emission regions in EIT images.

At higher levels, we used potential field extrapolations that we obtained with the potential field source surface (PFSS) routine in the solar software (SSW) package (see, e.g., Schrijver 2001; Schrijver \& De Rosa 2003). We used both EIT images and PFSS extrapolations as guidelines to infer where the magnetic field may be open and compared this with the outcome from UVCS data analysis. We point out that UVCS data are taken at altitudes high enough to be close to the source surface height (at least for the two highest positions of the slit), hence we expect the large-scale magnetic field there to be reasonably close to a potential field.

\section{Data analysis}

\subsection{Doppler dimming analysis}

The efficiency of the chromospheric radiation scattering process by a coronal ion is reduced when the plasma is moving, relative to the case of a static plasma, because the central wavelength of the scattering profile is Doppler shifted with respect to that of the line emitted from the solar disk. This is known as Doppler dimming (DD) effect, and its application as a diagnostic tool to measure the radial components of the outflow speeds in the corona has been extensively studied in the literature (see, e.g., Hyder \& Lites 1970; Withbroe et al. 1982; Noci et al. 1987; Noci \& Maccari 1999).

In the following, we briefly summarize the method (based on the DD effect) by which we determined plasma outflow speeds and electron densities. A more complete description is provided in Zangrilli et al. (2002) and Zangrilli \& Poletto (2012). Briefly, we recall that the intensities of the $\mathrm{H} \mathrm{I} \mathrm{Ly} \alpha$ and O VI doublet lines depend on the plasma density, the outflow speed, and the element abundance; other parameters such as the plasma electron temperature and the ion kinetic temperatures along and across the magnetic field direction play a secondary role. The data we used are the intensities of the H I Ly $\alpha$ line and the O VI doublet lines at 1031.9 and $1037.6 \AA$, which, provided the temperatures are known by some other means, are expected to allow us to infer the three unknowns: electron density, outflow speed, and element abundance. This is indeed the case whenever protons and oxygen ions flow at the same speed; while in $\mathrm{CHs}$ this probably does not occur (see, e.g., Cranmer 2009), we made this assumption at intermediate latitudes, hence we have enough parameters to solve the problem.

The coronal UV lines form by collisional and radiative excitations (see, e.g., Noci \& Maccari 1999). To estimate the plasma speed, we need to know by how much the radiative component of the coronal line intensities has been dimmed. To this end, we have to calculate the intensity of the line on the basis of a coronal model, starting with trial values of the plasma speed and electron density, and integrating the calculated local emissivities along the LOS on the basis of the profiles of the physical parameters prescribed by the model. When the calculated intensity does not reproduce observations, the plasma speed and electron density are changed until the intensity converges toward the observed value, hence identifying the correct plasma outflow speed and density.

For the values assumed for the plasma temperatures, we adopted the electron temperature radial profile given by Gibson et al. (1999) and the O VI kinetic temperature profile measured by Strachan et al. (2004), both obtained for a coronal streamer during a solar activity minimum. Parallel and normal (to the magnetic field) temperatures were assumed to have the same value. This is consistent with the results of Frazin et al. (2003), who claimed that kinetic temperature anisotropy in streamer regions, if any, starts above an altitude of $2.6 R_{\odot}$. Neutral hydrogen kinetic temperatures were evaluated from our own data set of H I Lyman $\alpha$ profiles, taking into account corrections for the instrumental profile function and the spectrometer slit width.

A further parameter we did not discuss so far is the solar disk intensity, which affects the intensity of the coronal radiative line components. H I Lyman $\alpha$ disk intensities have been measured in the time interval we analyze by the SOLSTICE experiment on the UARS satellite (see Woods et al. 2000). We point out that SOLSTICE measures the disk intensity from the same vantage point as Earth, while a coronal ion lying in the plane of the sky, directly above the AR, or in other words, in the most efficient geometrical scattering position, can be excited by a higher $\mathrm{H}$ I Lyman $\alpha$ flux originating from both the AR and the disk. A study of the AR contribution to the disk intensity has been made by Ko et al. (2002), who concluded that the AR contribution at the distances of the intermediate corona only amounts to a few percent of the total disk brightness. We took into account the small variation in the $\mathrm{H}$ I Lyman $\alpha$ disk brightness induced by the AR, adopting the value measured by SOLSTICE when the AR was at the central meridian, that is, six days before (for the west) or after (for the east) limb transit. Because there are no routine measurements of the $\mathrm{O}$ VI disk intensities, we used the values of 387.0 and $199.5\left(\mathrm{erg} / \mathrm{s} / \mathrm{cm}^{2} / \mathrm{sr}\right)$ for the 1031.9 and $1037.6 \AA$ lines, respectively, derived from UVCS disk observations taken on December 4, 1996 (see Zangrilli et al. 2002). The adopted line widths are taken from Warren et al. (1997).

\subsection{Si XII $520.7 \AA$ line analysis}

The Si XII doublet line at $520.7 \AA$, which is detected in the second-order spectra of the O VI channel, offers us the opportunity of analyzing the behavior of a low-FIP element line intensity along the UVCS slit. As we mentioned in the Introduction, the low-FIP element abundances are enhanced in the slow wind with respect to fast wind abundances, which are approximately photospheric. Unfortunately, from the $520.7 \AA$ Si XII line intensity, I(Si XII), and the electron density derived from the DD analysis alone, we cannot establish whether $I(\mathrm{Si}$ XII) changes across the 
AR because of variations in the $\mathrm{Si}$ abundance or in the electron temperature, or as a result of a combination of these factors. We discuss our results in Sect. 5.

We know that the Si XII $520.7 \AA$ line is collisionally excited, and its intensity is given by (see, e.g., Withbroe et al. 1982; Noci \& Maccari 1999)

$I(\mathrm{Si}$ XII $)=\frac{h v_{0}}{4 \pi} 0.83 A_{\mathrm{Si}} \int_{\mathrm{LOS}} R_{\mathrm{Si}^{+11}}\left(T_{\mathrm{e}}\right) n_{\mathrm{e}}^{2} C_{\text {coll }}\left(T_{\mathrm{e}}\right) \mathrm{d} x$,

where the $\mathrm{Si}^{+11}$ ionization ratio, $R_{\mathrm{Si}^{+11}}=n_{\mathrm{Si}^{+11}} / n_{\mathrm{Si}}$, is dictated by the ionization balance assumption and is a function of the electron temperature, $T_{\mathrm{e}} ; n_{\mathrm{Si}^{+11}}$ and $n_{\mathrm{Si}}$ are the $\mathrm{Si}^{+11}$ and silicon number density, respectively; $A_{\mathrm{Si}}=n_{\mathrm{Si}} / n_{\mathrm{H}}$ is the silicon abundance relative to hydrogen, $n_{\mathrm{H}} / n_{\mathrm{e}}=0.83$ is the hydrogen abundance relative to electrons, for a plasma composition with $10 \%$ of helium, $n_{\mathrm{e}}$ is the electron density; and $C_{\text {coll }}$ is the collisional coefficient of the transition, which is a function of $T_{\mathrm{e}}$.

Equation (1) can be written as

$I(\mathrm{Si}$ XII $) \propto\left[A_{\mathrm{Si}}\right]\left[n_{\mathrm{e}}^{2}\right] f\left(T_{\mathrm{e}}\right) L_{\mathrm{n}_{\mathrm{e}}^{2}}$,

where $\left[n_{\mathrm{e}}^{2}\right]$, the integration length scale $L_{\mathrm{n}_{\mathrm{e}}^{2}}$ and $f\left(T_{\mathrm{e}}\right)$ are usually evaluated around the temperature at which the line forms most strongly. In the following, we discard data acquired at the lowest slit position because the DD technique is more sensitive to the high-speed regime, and we discard data taken at slit positions 3 and 4 because counts in the line are often too few to guarantee a statistically significant result. Hence we use only data from slit position 2. For convenience, we write $I(\mathrm{Si} \mathrm{XII}) /\left[n_{\mathrm{e}}^{2}\right]$ below, which is proportional to $\left[A_{\mathrm{Si}}\right] f\left(T_{\mathrm{e}}\right)$ when the integration length scale $L_{n_{\mathrm{e}}^{2}}$ is constant. We are interested in changes across the AR of the $I(\mathrm{Si} \mathrm{XII}) /\left[n_{\mathrm{e}}^{2}\right]$ quantity, therefore we choose to give its profile along the slit, in arbitrary units normalizing to its mean value over the altitude interval -63 to $-54 \mathrm{deg}$, avoiding uncertainties in the calibration of UVCS second-order spectra.

\subsection{Potential field extrapolation}

Inferring the coronal magnetic field topology allows us to identify the sources of the outflows detected by the DD analysis, and, possibly, to locate them at lower levels. The potential field source surface (PFSS) extrapolation technique (see, e.g., Schrijver 2001; Schrijver \& De Rosa 2003) gives basic information on the magnetic field structure of the quiet corona. In particular, we used this approach to locate areas within the active region in which open magnetic field lines are rooted, assuming that the solar wind flows along open lines to eventually escape into interplanetary space. The PFSS package included in the SSW tree (Schrijver \& De Rosa 2003) relies on a database of solar surface magnetic field models, calculated with a sixhour cadence, obtained from SOHO/MDI. These magnetic field models are given for any heliographic latitude and Carrington longitude, and allow magnetic field lines to also be extrapolated at the time ARs are at the solar limb, as in the case of UVCS observations.

We extrapolated magnetic field lines in a longitude interval of \pm 10 deg across the plane of the sky in a latitude interval spanning from the equator to the south pole, thus covering the whole extent of AR 8100. The lines were traced on a grid uniform in latitude and longitude, without setting any treshold for the magnetic flux at the solar surface. In the following, we roughly assume that open lines originating from latitudes higher than -60 deg belong to the polar $\mathrm{CH}$ region.

\section{Results}

In this section we illustrate the results inferred from the DD technique described in Sect. 3.1 and from the analysis of the Si XII $520.7 \AA$ line, starting from the first limb transit of AR 8100, which occurred on October 26-27, 1997, to the last one, on February 27-28, 1998. The outflow speed results are shown as isocontour maps, obtained by interpolating the results of the DD analysis (see Fig. 2) as a function of latitude and heliocentric distance. Evidence for outflow channel candidates is provided by isocontour maps, dipping down, with respect to adjacent positions, toward lower altitudes. We required this to occur at several speed levels and at positions where open bundles of magnetic field lines, originating within the AR, are present. A further guide to identifying the AR outflow channels was obtained from the intensity ratio of the $\mathrm{O}$ VI doublet lines at 1031.9 and $1037.6 \AA, I_{\mathrm{O}} \mathrm{VI}-1031.9 / I_{\mathrm{O}} \mathrm{VI}-1037.6$. This diagnostic technique has been illustrated by many authors (see, e.g., Noci et al. 1987; Li et al. 1998), here it suffices to say that the intensity ratio is a decreasing function of the plasma speed, $v$, at least for $v<100 \mathrm{~km} \mathrm{~s}^{-1}$. Examples of the profiles of $I_{\mathrm{O}} \mathrm{VI}-1031.9 / I_{\mathrm{O}} \mathrm{VI}-1037.6$ along the UVCS slit are discussed in Sects. 4.2 and 4.3 at the times of the second and third AR limb transits. When the bundles of open lines clearly originated solely from the south $\mathrm{CH}$, (i.e., at southern latitudes roughly higher than $60 \mathrm{deg}$ ), we discarded the channel candidate from our analysis. The occurrence of open lines that project onto the same area and originate from both the $\mathrm{CH}$ and the $\mathrm{AR}$, as is often the case in channels at intermediate latitude, is discussed in Sect. 5.

The speeds and electron densities inferred from the DD analysis that belong to outflow channel candidates, which are tentatively classified as low-latitude and intermediate-latitude channels, are also given in separate diagrams (see Fig. 3). The error bars were estimated from the total counts statistics in the line intensites. The main source of error in the values inferred for the outflow speed and electron density is the statistical uncertainty in the intensity line ratio $I_{\mathrm{O}} \mathrm{VI}-1031.9 / I_{\mathrm{O}} \mathrm{VI}-1037.6$ (the H I Lyman $\alpha$ intensity is affected by a negligible error). We estimated the upper and lower limits in $v$ and $n_{\mathrm{e}}$ by setting the value of the ratio $I_{\mathrm{O}} \mathrm{VI}-1031.9 / I_{\mathrm{O}} \mathrm{VI}-1037.6$ to its highest and lowest value in the DD analysis, according to the Poissonian statistics of the intensities of individual lines. Owing to the non-linear relationship between the oxygen doublet intensity ratio and the outflow speed (see, e.g., Noci et al. 1987), values inferred from the DD study that are lower than about $50 \mathrm{~km} \mathrm{~s}^{-1}$, which are obtained mostly at low heliocentric distances, tend to be more uncertain than those relative to higher speed regimes, in spite of the more favorable count statistics. Typical outflow speed and electron density errors range within the intervals of 10-40 percent, and 2-10 percent, respectively. For a thorough discussion on the influence of DD model parameters on the final results we refer to Zangrilli \& Poletto (2012).

The contour maps of Fig. 4 describe the distribution and the evolution of the surface (photospheric) magnetic field strength of AR 8100 and the change in the orientation of the field polarities during Carrington rotations (CR) from 1929 to 1933. The data of these magnetograms are taken from the synoptic charts published by the Wilcox Solar Observatory ${ }^{1}$. The $\mathrm{CH}$ boundaries are also shown in Fig. 4a; data are taken from the synoptic maps of the He I $10830 \AA$ line equivalent width of the Kitt Peak telescope of the National Solar Observatory ${ }^{2}$.

\footnotetext{
http://wso. stanford.edu/synopticl.html

ftp://vso.nso.edu/kpvt/synoptic/
} 

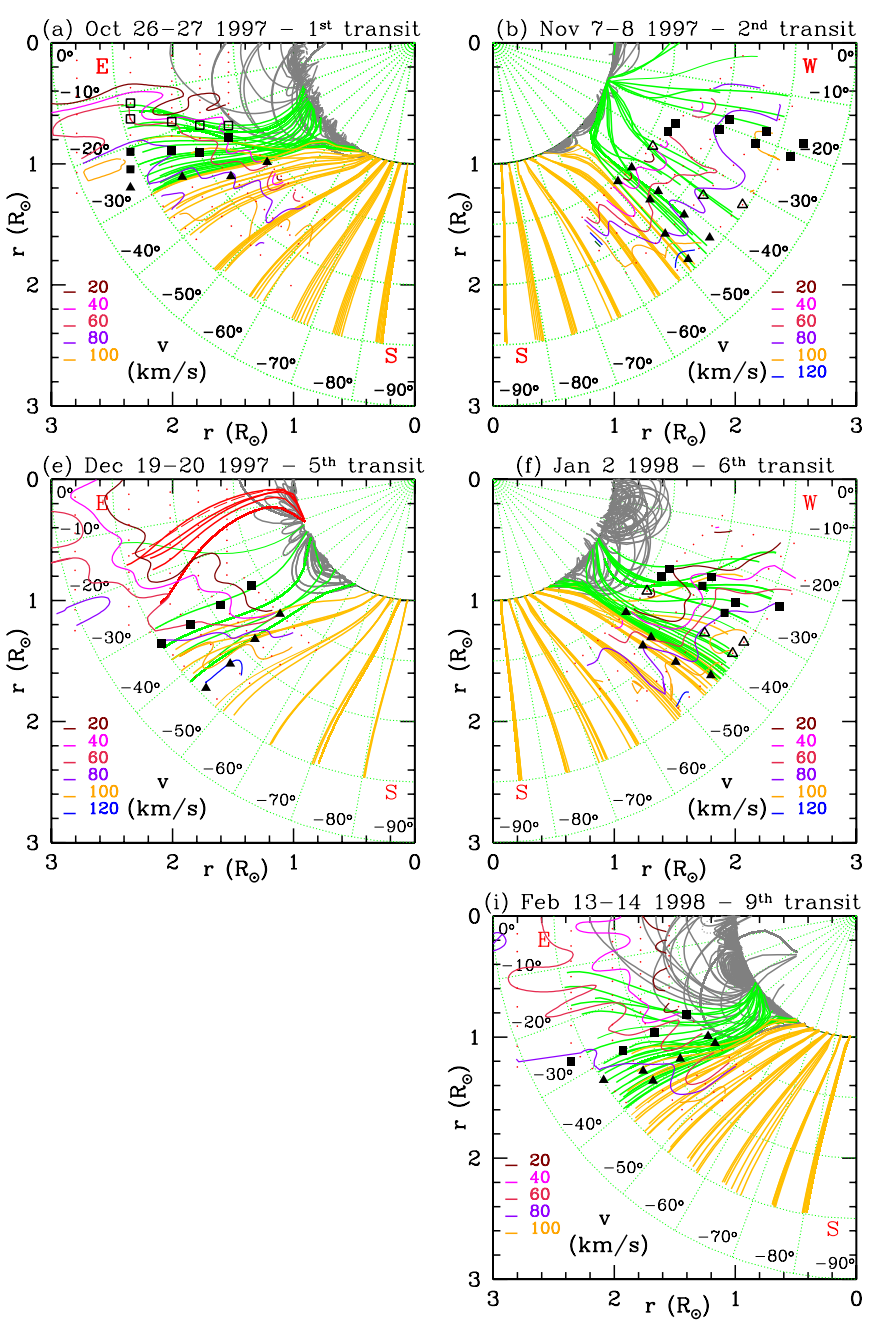

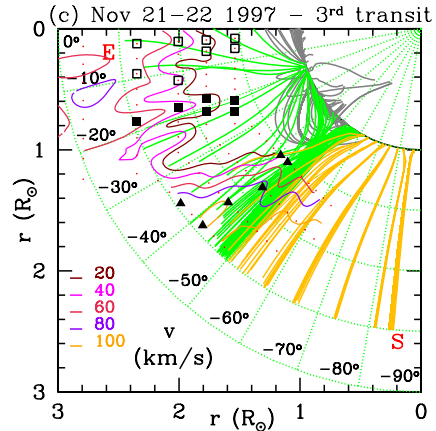

(g) Jan $16-171998-7^{\text {th }}$ transit
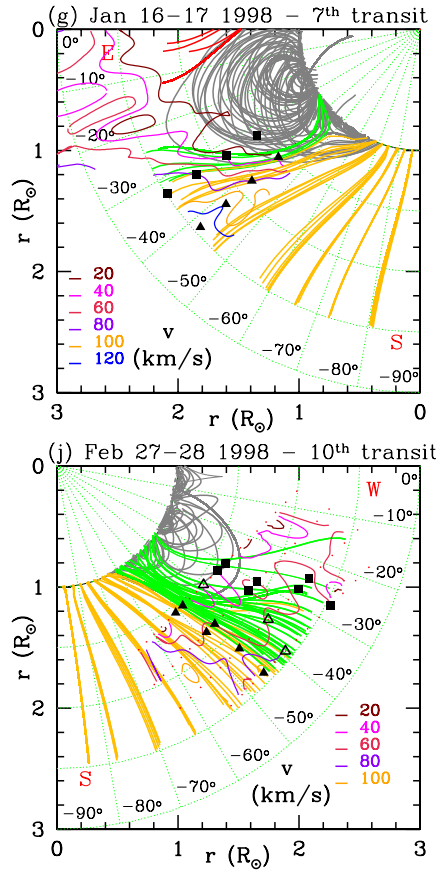

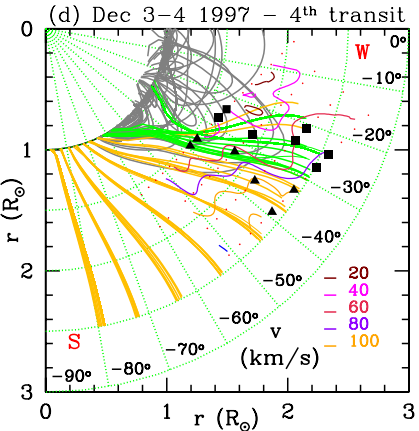

(h) Jan 29-30 $1998-8^{\text {th }}$ transit

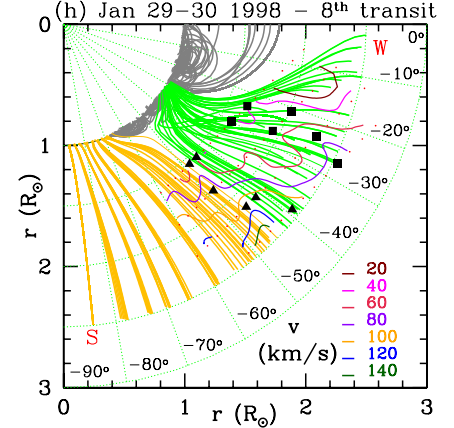

Fig. 2. Isocontour maps of the outflow speed for the ten limb transits of AR 8100. Open negative magnetic field lines originating from latitudes lower (higher) than $-60 \mathrm{deg}$ are shown in green (orange). In panels e) and g) positive open lines are also shown (red), originating from AR 8100 and from AR 8141 (in the northern emisphere), respectively. The UVCS slits are shown as a sequence of red dots that represent the individual bins. Full triangles (squares) identify data points within the intermediate- (low-)latitude outflow channel candidates. Additional outflow channels whose latitudes are slightly shifted equatorward with respect to the latitudes at which intermediate- and low-latitude channels are found are indicated with open triangles and squares.

We show in Fig. 3 the outflow speeds and densities for every limb transit of the AR and in Fig. 5 the profiles of $I(\mathrm{Si} \mathrm{XII}) /\left[n_{\mathrm{e}}^{2}\right]$ along the slit (set at position 2) for the AR west limb transits. Features for each limb passage are illustrated in individual subsection and discussed in Sect. 5.

\subsection{First limb transit}

The results from the DD analysis of the first limb transit, which occurred in the SE quadrant on October 26 and 27, 1997, are shown in Fig. 2a. Red dots indicate the location of the UVCS data used in our modeling; magnetic field lines obtained from PFSS extrapolations are labeled with different colors, gray (closed field lines), green (open), and orange (open, but originating from southern latitudes greater than -60 deg that pertain to the south polar $\mathrm{CH}$. Hints of three outflow channels appear in the intermediate corona at latitudes $-15,-25$, and $-35 \mathrm{deg}$; they are identified in panel (a) of Fig. 2 by dips in the contour levels of the speed map that occur at different altitudes where bundles of open lines are found. They originate in a loop system and in the boundary of the active region and the coronal hole
(AR-CH). Their positions correspond to local minima in the $I_{\mathrm{O}} \mathrm{VI-1031.9} / I_{\mathrm{O}} \mathrm{VI}-1037.6$ profiles. We classified two of them as low-latitude and intermediate-latitude channels: these are identified by full squares and full triangles, respectively in the map of Fig. 2a. A third outflow is indicated by open squares and apparently bifurcates from the low-latitude outflow at $1.7 R_{\odot}$ to then extend closer to the east equator. We note that the intermediatelatitude channel is also partially associated with open lines originating from the south polar $\mathrm{CH}$. These lines project onto the same areas as those rooted in the AR.

Figure 3a shows the speed profiles of the three outflow channels we found from the DD analysis. The low-latitude speed profile (solid squares) possibly attains values lower than those of the intermediate-latitude profile (solid triangles), particularly at distances smaller than $2 R_{\odot}$, while at higher altitudes the two profiles reach similar values. However, the relatively large errors do not allow us to draw any clear conclusion about possible differences in the speed regime between the two outflows. The secondary branch of the low-latitude channel (open squares) continues at relatively low speed values, also at distances larger than $2 R_{\odot}$. 

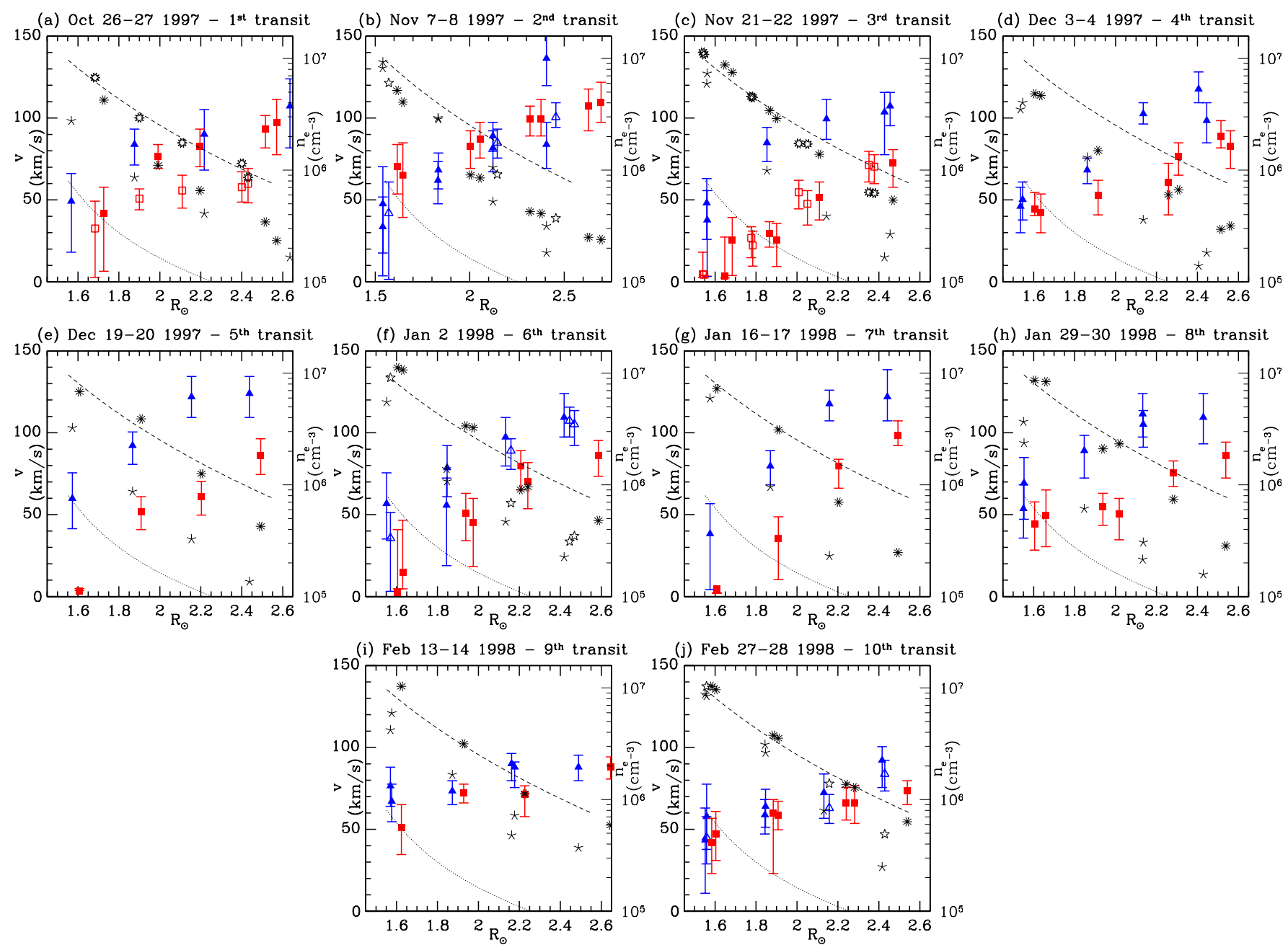

Fig. 3. Outflow speed and electron density vs. distance for the ten limb transits; the dotted and dashed curves are typical density profiles for a CH and a streamer, respectively (see Guhathakurta et al. 1999; Gibson et al. 1999). The solid blue triangles and the solid red squares are the intermediate- and low-latitude outflow speeds, respectively. The five- and ten-point stars represent the electron density values for the intermediateand low-latitude outflows, respectively. The error bars in the electron density are not shown as they are barely distinguishable in a logarithmic plot; see text for a discussion about the error estimate for $v$ and $n_{\mathrm{e}}$. Analogously to Fig. 2, open triangles and squares refer to the outflow speeds in secondary intermediate- and low-latitude channels; the relative electron densities are given as open ten- and five-point stars.

The low-percentage errors in the calculated electron densities allow us to state that the three density profiles (see Fig. 3a) are different: in particular, two profiles are intermediate between typical streamer and $\mathrm{CH}$ profiles, and the third one, pertaining to the equatorial region, is more similar to typical streamer values. Moreover, the highest latitude channel appears to show a mixture of plasma from the $\mathrm{AR}$ and the polar $\mathrm{CH}$.

The panels of Fig. 6 show the west and east side of AR 8100, as the AR moves from the east to the west limb passage. Moreover, the gradual rotation of the AR polarities occasionally brings different parts of its structure into the foreground. These parts appear to consist of three loop families. The first system is closest to the equator and consists of slanted loops, which are seen neither exactly face-on nor edge-on during the first transit. The second family appears at medium latitudes as a fainter and apparently higher reaching system than the first. It gradually brightens with time and evolves to a face-on orientation owing to the AR rotation, clearly revealing a fan-loop structure (see Fig. 6e). The third family is located at higher southern latitudes and consists of several bright features whose geometry is initially not easy to interpret. They are connected in the $\mathrm{N}-\mathrm{S}$ direction.

After the solar rotation dragged the AR close to the central meridian, where better magnetic field data could be acquired, multiple episodes of flux emergence were observed. The AR produced some 35 flares and 25 CMEs at this location during its first transit (see Green et al. 2002). Although the high variability makes any comparison between extreme ultraviolet (EUV) morphology and isocontour maps rather uncertain, we searched EIT images for areas of weaker EUV emission within the UVCS FOV because long-lived upflows have been recognized from Hinode/EIS analyses to correspond to low-intensity regions (e.g., Doschek et al. 2008; Del Zanna 2008; Hara et al. 2008; Démoulin et al. 2013). The EIT $195 \AA ̊$ A image in Fig. 6a clearly shows the occurrence of a weaker emission area in between the first and second loop system (latitude $\approx-24 \mathrm{deg}$ ) and, analogously, of a wide gap at the southern boundary of the second system at about -43 deg latitude. These positions nicely correspond to the region from which open magnetic field line bundles originate and are then superposed onto the channels in 

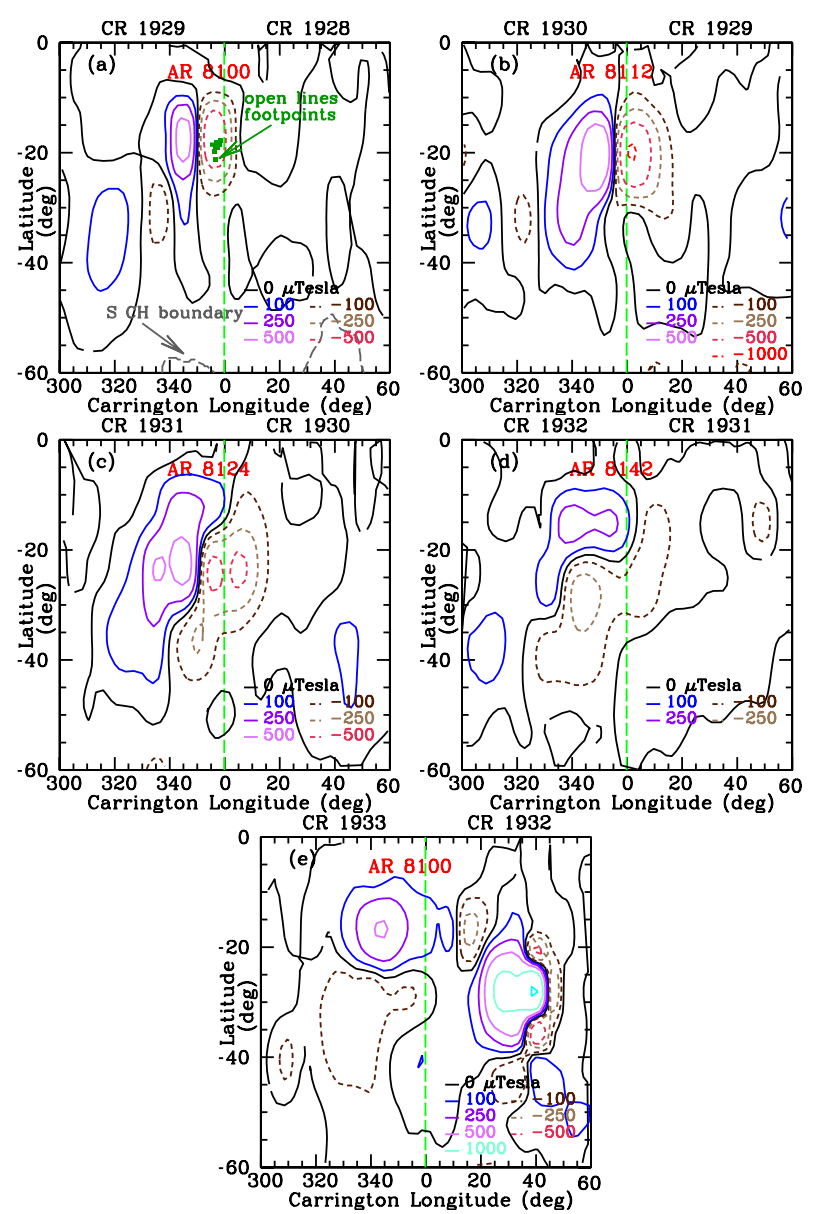

Fig. 4. Photospheric magnetic field distribution around AR 8100, taken during Carrington rotations from 1929 to 1933, adapted from the synoptic maps provided by the Wilcox Solar Observatory. The neutral field line is drawn as a black solid line, while positive and negative contours are drawn as colored solid and dashed lines. The greenish squares in panel a) are the footpoints of the open magnetic field lines. The $\mathrm{CH}$ boundaries are based on He I $10830 \AA$ A line observations from the Kitt Peak telescope and are shown as a gray long-dashed contour.

the outflow map. The positions of the weaker emission areas that we identify in the EIT images of the ten AR limb transits, and that we claim to be the low corona counterparts of the outflows we detected in the intermediate corona with the DD analysis, are indicated with red arrows in the panels of Fig. 6.

The clues we collected in the first AR 8100 limb transit that are in favor of the occurrence of outflow channels in regions of open field lines and where the EIT images show low-density areas, would be corroborated if the following limb transits showed a similar behavior. We therefore analyzed successive limb passages of AR 8100 to provide further support of the evidence provided so far.

\subsection{Second limb transit}

During the second AR limb transit, AR 8100 was fairly active: the shearing motions have been highlighted by Green et al. (2002), and according to the LASCO CME catalog ${ }^{3}$, on November 8 a CME spanning 76 degrees occurred at $\mathrm{PA}=$ $271 \mathrm{deg}$ at 08:59 UT, and two CMEs occurred on the previous

\footnotetext{
3 http://cdaw.gsfc.nasa.gov/CME_list/
}
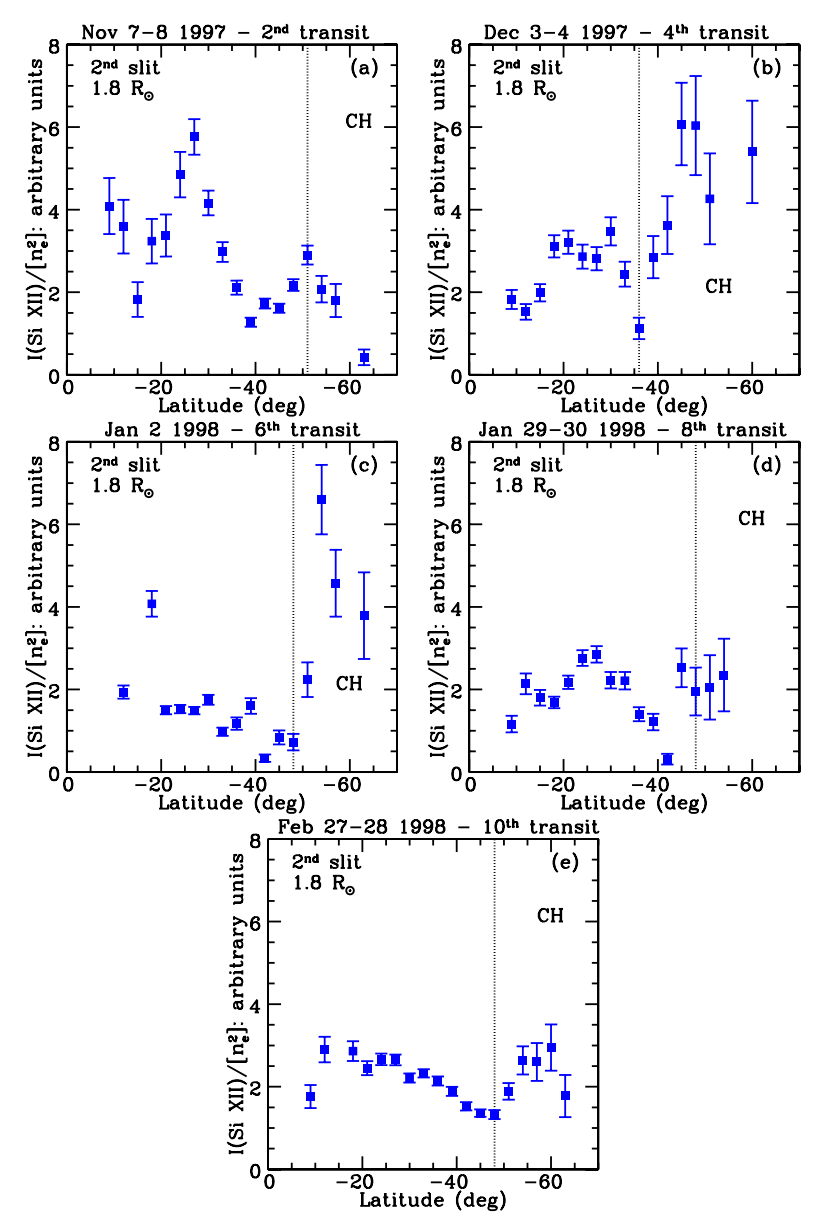

Fig. 5. Profile along the slit of the normalized (see Sect. 3.2) quantity $I(\mathrm{Si} \mathrm{XII}) /\left[n_{\mathrm{e}}^{2}\right]$, for the limb transits $2,4,6,8$ and 10 . The equator corresponds to $0 \mathrm{deg}$, the south pole to $-90 \mathrm{deg}$. The vertical dotted line defines the boundary with the south polar $\mathrm{CH}$.

day at PAs $270 \mathrm{deg}$ and $278 \mathrm{deg}$, respectively. This shows that the magnetic configuration of the region experienced disruptions, although no event is recorded on November 9 . We mention that a X9.4 flare occurred on November 6 at 11:49 UT, followed by a halo CME at 12:10 UT, whose source has been identified in the AR 8100 site.

Analogously to the first transit, the outflow speed map in Fig. $2 b$ is characterized by three systems of channels above the AR at latitudes $-20,-35$, and $-45 \mathrm{deg}$; two of them appear to belong to the intermediate-latitude (solid and open triangles) and the third to the low-latitude family (solid squares).

Figure $3 \mathrm{~b}$ shows the outflow speed and electron density profiles of the low- and intermediate-latitude channels: the properties of the outflows appear indistinguishable in outflow speed and electron density. Figure 7 a shows the profile of the $\mathrm{O}$ VI doublet line intensity ratio, $I_{\mathrm{O}} \mathrm{VI}-1031.9 / I_{\mathrm{O}} \mathrm{VI}-1037.6$, along the UVCS slit at the time of the second limb transit of the AR in the four different positions of the UVCS slit at PA $=235 \mathrm{deg}$. It is obvious from the plot that the $\mathrm{O}$ VI line ratio is lowest at the high outflow channels.

The magnetic PFSS extrapolation is characterized by three loop systems with open field lines originating far apart on the east side (see Fig. 2b). The open magnetic field lines from the AR site define reasonably well the outflow lanes we found on the basis of the criteria we established. The most relevant difference with respect to the first transit concerns the topology of the open magnetic field lines, which clearly appear to originate 

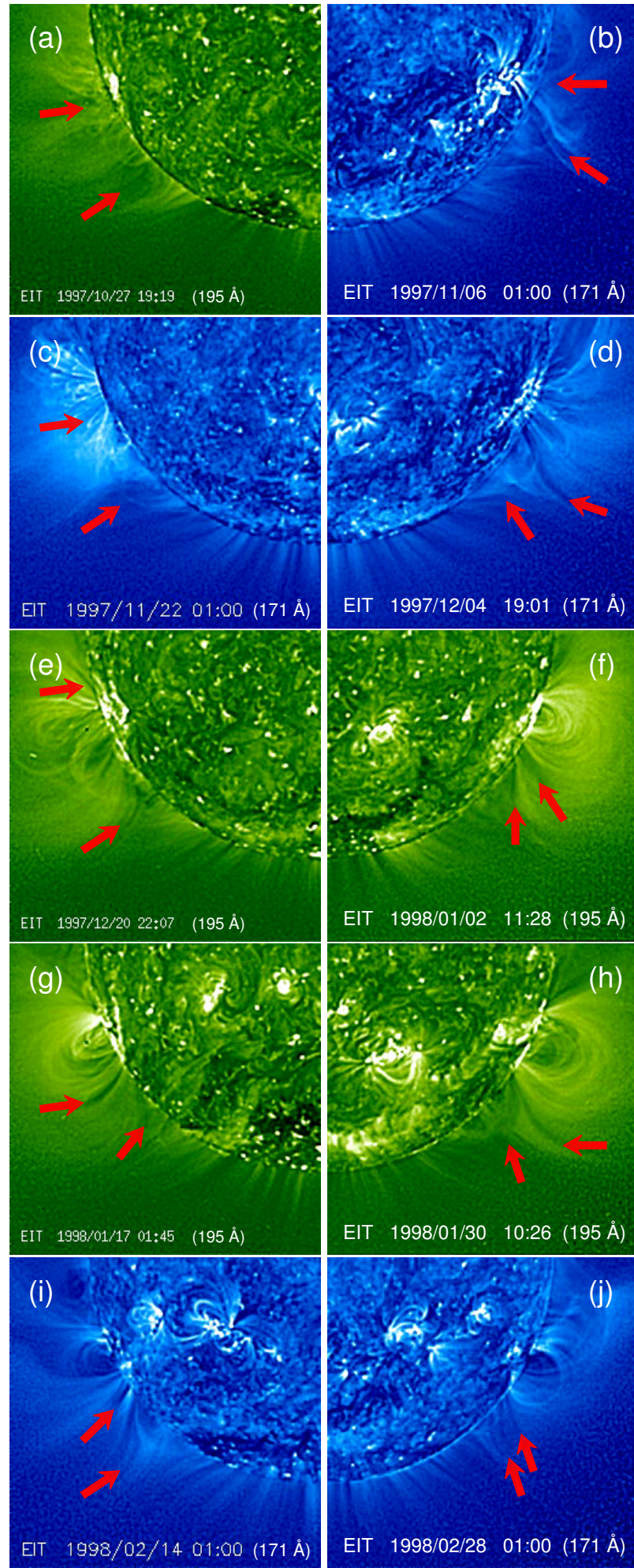

Fig. 6. EIT $171 \AA$ and $195 \AA$ A wavelet-enhanced images, acquired during the AR limb transits. In the panels the red arrows point to weak emission regions, which we identify as the low corona counterparts of the low- and intermediate-latitude outflow channels that we infer from the DD analysis of UVCS data. We considered EIT $195 \AA$ A images when the $171 \AA$ channel data were not available. For the second limb transit (panel b), we had to consider images taken on November 6 because no EIT data were available for November 7 and 8 .

from a common region inside the AR, as shown in Fig. 2b. The AR magnetic field map for CRs 1928/1929 is also presented in
Fig. 4a, where all the footpoints of the open field lines (greenish squares, corresponding to the green open lines of Fig. 2b) lie within the same portion of negative polarity of the AR and are far from the AR-CH boundary (gray long-dashed contour).

The configuration of the AR at its second west limb passage is presented in Fig. 6b, which shows a wavelet-enhanced EIT $171 \AA$ image taken on November 6, 1997, one day before the time interval we considered in the DD analysis, because no wavelet EIT images are available on November 7-8. Figure 6b clearly shows a dark lane at the southern latitudes $\approx-40$ and -25 deg (both marked with red arrows), matching the positions of the outflow channels and of the open field lines in the PFSS extrapolation (see Fig. 2b).

A different view of the field line geometry is shown in Fig. 8, from a vantage point on the west limb of the Sun, that is, above the AR at the time it crosses the west limb (the view is rotated by 90 deg with respect to that of Fig. 2b), where the green open lines originate from the $\mathrm{AR}$, and the orange lines from the $\mathrm{CH}$. The west AR side is characterized by a fan-loop structure, while from the east side the closed lines connect the main polarity to the network magnetic field. The bundle of open field lines that have been shown to overlie the outflow channels inferred from the DD analysis in corona originates in between these two loop structures. Recent studies revealed that fan-loop structures are often associated with quasi-separatrix layers (QSLs; see, e.g., Schrijver et al. 2010). Baker et al. (2009) identified the QSLs that separate closed field lines, where the magnetic field connectivity experiences a drastic change, as regions from which AR outflows may originate. Although detailed calculations are required to find the possible location of QSLs in the AR magnetic field topology, the magnetic field structure shown in Fig. 8 indeed suggests the occurrence of QSLs at the border separating the open magnetic field lines from the fan-loop system, hence enforcing our claim of having identified AR outflows in the intermediate corona.

Panel a of Fig. 5 shows the profile of $I(\mathrm{Si} \mathrm{XII}) /\left[n_{\mathrm{e}}^{2}\right]$ across the UVCS slit at the time of the second limb transit of the AR and an altitude of $1.8 R_{\odot}$ : the profile shows two peaks at latitudes $\approx-25$ and $\approx-50 \mathrm{deg}$, which coincide with the positions of the two main outflows identified in the DD analysis (see Fig. 2b). Under the hypothesis that the electron temperature, hence the function $f\left(T_{\mathrm{e}}\right)$, is constant along the UVCS slit (see the discussion in Sect. 3.2), the variation in the ratio $I(\mathrm{Si} \mathrm{XII)}) /\left[n_{\mathrm{e}}^{2}\right]$ can be explained by invoking a silicon enrichment in the coronal plasma at the positions of the two peaks, which is where we detected slow wind outflows. This interpretation is also valid when the temperature in the channel is lower than the temperature of adjacent regions because $f\left(T_{\text {e,channel }}\right)<f\left(T_{\mathrm{e}, \mathrm{AR}}\right)$ (according to the ionization balance calculations given in the literature, see, e.g., Arnaud \& Rothenflug 1985, we have the maximum of Si XII fraction for $T_{\mathrm{e}} \approx 2 \times 10^{6} \mathrm{~K}$, which is above the electron temperature regime we expect at these altitudes; see the discussion in Sect. 5) and a higher Si abundance would be required to account for our results. This means that the plot would be consistent with the enrichment of the low FIP elements found by other authors in AR outflows, as mentioned in Sect. 1. At the altitude we examine, however, the Si XII ion is very likey frozen-in, and we have no means to know the temperatures at which this phenomenon occurs in the channels and in the AR. Because (see Sect. 3.2) the Si XII abundance is extremely sensitive to temperature variations, we cannot rule out the hypothesis that our results have been dictated by different temperature regimes along the UVCS slit and not by changes in the Si abundance. 

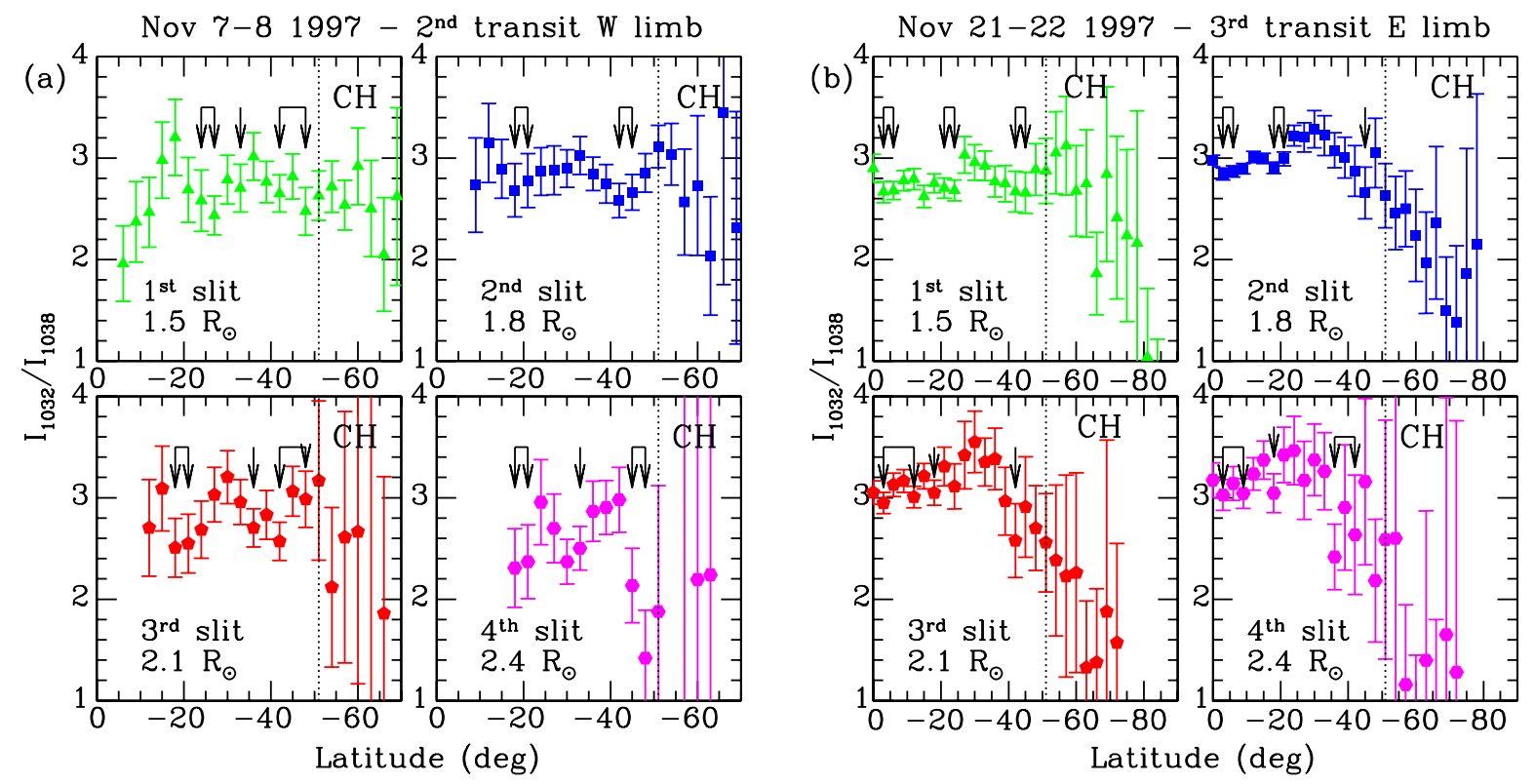

Fig. 7. Profiles of the O VI doublet intensity ratio along the UVCS slit at four slit positions from data taken at PA = 225 deg (panel a)), and $\mathrm{PA}=135 \mathrm{deg}$ and $\mathrm{PA}=90 \mathrm{deg}($ panel $\mathbf{b})$ ) for the second and third limb transit, respectively. The positions of the AR outflows are shown by arrows, and the vertical dotted line defines the boundary with the south polar $\mathrm{CH}$.

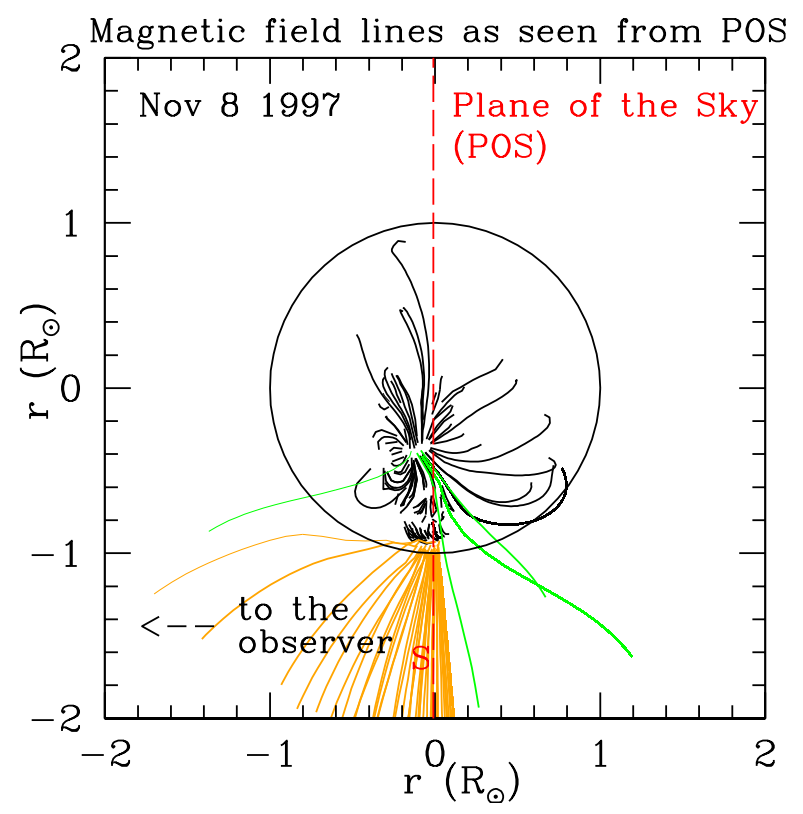

Fig. 8. PFSS extrapolation above the AR at the second limb transit, as seen from an observer directly above the AR, on the west limb of the Sun. The arrow points to the position of UVCS at the time of observations.

\subsection{Third and fourth limb transits}

The third limb transit of the AR occurred on November 21-22, 1997, when the AR was still very active and changing features appeared at different times. The shearing motion of the AR gradually brought the negative polarity closer to the south polar $\mathrm{CH}$ (see Fig. 4b), which is the reason for the edge-on view of some of the fan-loop system that makes up the western part of the AR. In the EIT image shown in Fig. 6c, the three-loop configuration is now less evident, and the dark lanes are probably partially covered by the large central loop system. Nonetheless, in Fig. 2c plasma regions at relatively high outflow speed are distinguishable at latitudes -20 and $-40 \mathrm{deg}$, supporting results obtained in the preceding limb transits. Another somewhat less evident outflow can be distinguished just below the east equator, associated with a few open lines. It reaches latitudes of about $-10 \mathrm{deg}$. The $I_{\mathrm{O}} \mathrm{VI-1031.9} / I_{\mathrm{O}} \mathrm{VI}-1037.6$ profiles in Fig. $7 \mathrm{~b}$ confirm the system of three outflows from the AR (two low-latitude and one intermediate-latitude channels), at their positions derived from the map in Fig. 2c.

Green et al. (2002) made linear force-free extrapolations of the coronal field to match low-lying Yohokh SXT loops for five rotations of AR 8100. In their Fig. 5 the authors present the extrapolation for November 29, 1997 and show that an $\alpha$ value of $0.94\left(10^{-2} \mathrm{Mm}^{-1}\right)$ best reproduces the X-ray Yohkoh loops. Although obviously different from the potential field we computed, the three-loop system is still apparent in the Green et al. (2002) extrapolation, which supports the results of our technique, which compares UV features at high heliocentric altitudes with potential coronal fields.

The fourth limb transit of the AR, on December 3-4, 1997, occurred at a time when its configuration was quite clear in EIT maps. Figure 6d shows the EIT $171 \AA$ A map on December 4, 1997, where the three-loop system configuration is clearly visible. It is obvious that the AR consists of a northern, southern, and central loop system. The central system originates in the western part of the AR and is now seen edge-on, leaving a clearly defined dark lane from the site of the AR in the foreground and a less evident lane from the AR-CH boundary. Two bright ribbons, oriented along the N-S direction, whose separation increases with increasing latitude, are easily distinguished in the EIT image. The dark lane seems to originate from these two ribbons. They are visible in the first two west transits in Figs. 6b and d and are probably covered by the central loops when they are at the east limb.

The coronal PFSS extrapolation for the same day (see Fig. 2d) shows open fied lines originating from latitude $-25 \mathrm{deg}$ and from the boundary of the AR with the south $\mathrm{CH}$, and extending in the intermediate corona approximately along the radial directions at -25 and $-35 \mathrm{deg}$. A partial overlay of open 
magnetic lines from the south $\mathrm{CH}$ with those coming from the AR affects the intermediate-latitude outflow. The agreement between the occurrence of the obvious dark lanes in the EIT image with the high-speed lanes in the UVCS outflow speed maps and the occurrence of open field lines in Fig. 2d appears here more strongly than in previous limb transits.

The corresponding magnetic field configuration is detected in the Wilcox Solar Observatory synoptic map for CR 1930, where the northernmost polarities of the AR are adjacent (see Fig. 4b). The map also accounts for the occurrence of large-scale trans-equatorial loops joining the south AR we analyzed and the $\mathrm{AR}$ at the limb in the northern hemisphere, which is partially visible in the EIT map. This configuration results in face-on transequatorial loops and edge-on central loops, separated by darker lanes, giving us a good opportunity of identifying the source region of high-speed lanes.

The profile of the ratio $I(\mathrm{Si} X I I) /\left[n_{\mathrm{e}}^{2}\right]$ during the fourth transit is shown in Fig. 5b, where we can recognize two peaks at -20 and $-30 \mathrm{deg}$, more or less corresponding to the channel positions in Fig. 2d, and a higher maximum between -40 and $-45 \mathrm{deg}$, which corresponds to open lines from latitudes higher than $-60 \mathrm{deg}$, however. We must add that a CME occurred on December 5, 08:27 UT, PA = $275 \mathrm{deg}$, spanning 98 degrees, and PFSS extrapolation might not accurately represent the magnetic field topology during the fourth limb transit.

\subsection{Fifth and sixth limb transit}

The fifth passage occurred on December 19-20, 1997, when the AR configuration appeared to evolve toward a morphology consisting of only two loop systems, as can be deduced from Fig. 6e. This is probably to be ascribed to the AR progressive rotation, which oriented the central loop system to a more face-on angle. In addition to the evident channels at -32 and $-45 \mathrm{deg}$, a hint of a third channel is visible in Fig. 2e at latitude $-25 \mathrm{deg}$, which is probably associated with positive open lines from the northeast side of the AR and is plotted as red lines in the same figure. In the EIT images the two ribbons of the AR, which during the fourth transit where N-S oriented, now appear to rotate (see Fig. 6e), following the AR polarity evolution (see also Fig. 4c, showing the AR polarity rotation).

The sixth AR limb transit occurred on January 2, 1998, and has been the subject of the Zangrilli \& Poletto (2012) paper. We refer to this paper for detailed information on that limb transit. Here it suffices to recall that the authors concluded that a narrow channel at a latitude of $-45 \mathrm{deg}$ was the site of high-speed outflows, originating from the southernmost edge of the AR. This is visible in Fig. $2 \mathrm{f}$ (solid triangles), where we also show a second intermediate-latitude outflow from the AR (open triangles at $-35 \mathrm{deg}$ ) and a low-latitude outflow, between -20 and $-30 \mathrm{deg}$ (solid squares). The three channels are associated with three distinct bundles of open lines, two originating from latitudes between -30 and $-40 \mathrm{deg}$ and one from the AR-CH boundary. We point out that in the $-45 \mathrm{deg}$ channel open field lines both from the AR and from the south polar $\mathrm{CH}$ contribute to the observed outflow, analogously to the outflows we found in similar circumstances in other limb transits. We recall that the speed map in the present work has been made by averaging over a latitudinal width of three degrees, while Zangrilli \& Poletto (2012) averaged over the original data to obtain a constant spatial resolution of 105 arcsec along the slit for all the four slits of the dataset. This may account for minor differences between plots in this and the previous paper.
The configuration of the AR in previous transits and its configuration in this passage is changed remarkably: this is illustrated in Fig. 4c, where the WSO magnetogram of AR 8100 for CR 1931 shows the AR polarity rotation at its early stages, and Fig. 6f, where we show a large face-on loop system, with little evidence of the loop families imaged in earlier transits.

In Fig. $5 \mathrm{c}$ we show two maxima in the $I(\mathrm{Si}$ XII $) /\left[n_{\mathrm{e}}^{2}\right]$ profile, as expected on the basis of the well-defined low-latitude outflow, which is now quite distinct from the channel visible at $-45 \mathrm{deg}$. A small peak at -38 deg possibly accounts for the third outflow, as shown in the speed map of Fig. $2 f$.

\subsection{Seventh and eighth limb transits}

The seventh and eighth AR limb transits occurred on January 16-17 and 29-30, 1998, respectively. The AR configuration is similar to that of the preceding sixth transit (see Figs. $6 \mathrm{~g}$ and $h$ ), and it is dictated by the E-W alignement of the neutral line, as shown in Fig. 4d. Figure $6 \mathrm{~h}$ shows that the AR ribbons now appear almost E-W oriented. This is an especially quiet time for AR 8100: no flare originated in the region until January 22. The outflow speed maps are shown in Figs. $2 \mathrm{~g}$ and $\mathrm{h}$.

In the seventh transit we probably have only a low-latitude channel (the intermediate-latitude channel is largely covered by open lines from the south polar $\mathrm{CH}$ ). The red positive open lines, partially visible in the equatorial side of Fig. $2 \mathrm{~g}$, pertain to AR 8141, which is in the northern hemisphere, and possibly account for the humps and dips in the speed map at equatorial latitudes. In the eighth passage the intermediate-latitude outflow is perhaps recognizable again, and only partially to be ascribed to open lines originating from the south polar $\mathrm{CH}$, as demonstrated by the PFSS extrapolation in Fig. 2h. This also agrees with the absence of a clear intermediate-latitude peak of the ratio $I(\mathrm{Si} \mathrm{XII}) /\left[n_{\mathrm{e}}^{2}\right]$ in Fig. $5 \mathrm{~d}$; analogously, the low-latitude maximum is barely distinguishable in a globally flattened profile.

\subsection{Last two transits}

The last two transits of AR 8100 occurred on 13-14 and 2728 February, 1998. After that time, the dispersal of the magnetic field in the network between growing nearby young ARs, AR 8162 on the east side and AR 8156 on the west side, makes AR 8100 barely identifiable (see Fig. 4e). In Figs. 2i and $\mathrm{j}$ the outflow maps pertaining to transits 9 and 10 are shown. The last period of the AR life is characterized by two remarkable facts: the flattening of the speed profiles in both the low- and intermediate-latitude outflows, and the flattening of the $I(\mathrm{Si} \mathrm{XII}) /\left[n_{\mathrm{e}}^{2}\right]$ profile (see Fig. 5e). Although the intermediate-latitude outflow still persists (two channels can be distinguished), the magnetic field of the AR negative polarity is weakening, as demonstrated by the map shown in Fig. 4e.

\section{Discussion and conclusions}

The purpose of this work is to analyze the evolution of AR outflows detected by the SOHO/UVCS spectrometer in the intermediate corona throughout the lifetime of NOAA AR 8100. The observations covered a period of four months from the end of October 1997 to the end of February 1998. Previous works about AR outflows evolution (e.g., Démoulin et al. 2013; Culhane et al. 2014) mainly focused on the time interval of the AR solar disk crossing, or took into account only a single AR limb transit (Zangrilli \& Poletto 2012). 
Our present study provides direct evidence that channels of coronal outflow originate from AR 8100, which appear at the very beginning of the AR life, and persist throughout the entire AR evolution. We observed at least two spatially distinct types of outflow channels: low-latitude outflows, associated with bundles of open magnetic field lines radially extending roughly from within the AR, and intermediate-latitude outflows, which, although detected close to the $\mathrm{CH}$ boundary, are often clearly associated with open field lines whose footpoints lie in the AR or in its immediate neighborhood. The evolution of the morphology and outflow locations in the corona is illustrated in Fig. 2, from which we conclude that a two-channel pattern roughly persists throughout the whole AR evolution, with minor changes in the latitudes at which the channels appear.

Our data do not allow us to show how far the outflows can be identified: do they really reach outer interplanetary space? ACE slow wind observations confirm what we proved out to $\approx 2.5 R_{\odot}$ : AR 8100 , during CR 1929, has been recognized as source of low-speed solar wind and as a site of open magnetic field lines by Rust et al. (2008) (see the synoptic chart shown in Fig. 6 therein), who traced back the slow wind observed by ACE at 1 AU to AR 8100.

The speed vs. heliocentric distance profiles in the channels are shown for each limb transit in Fig. 3. The speed profiles appear relatively flat early in the AR life; conversely, during the central part of the AR evolution, they reveal a more pronounced acceleration and finally tend to level off with the AR age. Both outflow speed and electron density appear to have different profiles in the two channels in several transits of the AR, as shown in Fig. 3. Electron densities in the low-latitude channels often tend to be higher than densities in the high-latitude channel, while the opposite occurs for the outflow speed profiles.

However, the exceptions represented by the second and possibly, although with a lower degree of evidence, by the first limb passage (see Figs. 3a and b), where the density profiles are almost indistinguishable, lead us to hypothesize that in some case, data may have been contaminated at intermediate latitudes by plasma from the south polar $\mathrm{CH}$. It is difficult to estimate how large this contamination might be, hence to draw quantitative conclusions about physical differences between the two types of outflow channels, that, as we pointed out, are likely to share a common origin (see, e.g., the PFSS extrapolations).

As we discussed in Sect. 3, in some cases (e.g., during the second limb transit) the PFSS extrapolation suggested that the two types of outflows we found (low-latitude and intermediatelatitude) might originate from a single portion of the AR, as some of the open field lines from the AR sharply bend toward the south polar $\mathrm{CH}$ boundary and then expand radially at the locations of intermediate latitude outflow channels. In some cases, open field lines from the south polar $\mathrm{CH}$ are clearly detected, for instance, during limb transits 6 and 8, which project onto the plane of the sky above the intermediate-latitude channel. The speed and density values deduced from the DD analysis in these cases might be an average of the two outflow regimes, that from the AR and that from the $\mathrm{CH}$. It is possible that the south polar $\mathrm{CH}$ open field lines physically come close together in the open bundles from the AR, as during the AR evolution the magnetic field topology changes and the AR rotation brings the $\mathrm{CH}$ boundary closer to the area of the AR from which the open field lines originate.

In this context, it is interesting to note that when in the intermediate-latitude outflow channels there is less superposition of $\mathrm{CH}$ and $\mathrm{AR}$ open field lines, we find that the outflow speed and density profiles appear indistinguishable from the low-latitude channels (see, e.g., Fig. 3b, which summarizes the results of the second limb transit). This result supports the hypothesis that all outflow channels we found have the same origin in the AR, and differences in the speed profiles originate from a contamination with $\mathrm{CH}$ outflows.

As we mentioned in Sect. 4.2, the AR structure from $\mathrm{SOHO} / \mathrm{EIT}$ images and from its magnetic field topology, as inferred from PFSS extrapolation, suggest that the origin of the outflows we detect in the intermediated corona is inside the negative AR polarity, at the boundary with a fan-loop system. This configuration is similar to other cases described in the literature (see Schrijver et al. 2010; Baker et al. 2009), in which QSLs in the vicinity of fan-loops and open magnetic field lines have been associated with AR outflows detected in the low corona.

A direct comparison of the speed values we derived here with measurements of AR outflow speeds in the literature cannot be done because we analyzed data at intermediate corona altitudes, whose speed regime has only been probed by UVCS with the DD technique. On average, the speed profiles radially accelerate from about 50 up to $150 \mathrm{~km} \mathrm{~s}^{-1}$ from 1.5 to $2.5 R_{\odot}$. This suggests that the AR outflows we observed have initial speeds lower than $50 \mathrm{~km} \mathrm{~s}^{-1}$, which is lower than the values we could expect for the high-speed component seen by Hinode/EIS (see Tian et al. 2011b) at lower altitudes. We may fail to observe the high-velocity component described by Tian et al. either because the high-speed AR outflows are episodic, transient, or quasiperiodic (see De Pontieu \& McIntosh 2010; Tian et al. 2011a) and UVCS only measures an average speed value over a two-day interval or because the three-degree spatial averaging we adopted is too coarse and they are smeared out.

The purpose of the analysis of the second-order Si XII $520.7 \AA$ line was to determine whether it might support the hypothesis that the outflows detected by UVCS in the intermediate corona are a component of the slow wind. The profiles of the quantity $I(\mathrm{Si}$ XII $) /\left[n_{\mathrm{e}}^{2}\right]$ along the UVCS slit, shown in Fig. 5, are characterized, especially during the early stages of the AR life, by two peaks at latitudes that fairly well match those of the AR outflows. This seemingly supports our claim; the strong dependence of the Si XII abundance on temperature and the lack of information on the precise profiles of temperature vs. height in the channels and in the AR allow us only to state that our results are consistent with the hypothesis of an enhancement of low FIP elements in slow wind, however. We found a general tendency of levelling-off of the $I(\mathrm{Si} \mathrm{XII}) /\left[n_{\mathrm{e}}^{2}\right]$ profiles across the UVCS slit at the end of the AR life, in agreement with the weakened evidence for outflow channels during the last two limb transits.

Evidence has been obtained in the low corona by Doschek et al. (2008), for example, for lower electron temperatures inside the AR outflows $\left(1.2-1.4 \times 10^{6} \mathrm{~K}\right)$, with respect to the surrounding closed corona. The Si XII curve of the ionization balance (see, e.g., Arnaud \& Rothenflug 1985) peaks at about $2 \times 10^{6} \mathrm{~K}$, well above the values estimated by Doschek et al. (2008). Under the hypothesis that $T_{\mathrm{e}}$ inside the AR outflows is maintained at values lower than the surrounding corona at the altitudes of UVCS observations, we would expect a decrease in the $I(\mathrm{Si} \mathrm{XII}) /\left[n_{\mathrm{e}}^{2}\right]$ profiles across the AR, which is the opposite of what we obtained. For this reason, we could speculate that we observe an effect of silicon abundance enhancement, the electron temperature possibly giving a negligible contribution. Moreover, because silicon is a low-FIP element, this would be consistent with the expectations for slow wind flows. The limited information we can obtain from our data prevents us from drawing any conclusion about a possible enhancement of the abundance of 
low-FIP elements in the outflow channels, leaving a clearer interpretation to future studies.

In conclusion, we have shown that ARs contribute throughout their lifetime to the slow wind. Whether their contribution is time dependent on short timescales cannot be determined from this analysis. On the other hand, our work provides strong support to the claim by Démoulin et al. (2013) that the upflow driver acts over a prolonged time, here shown to be on the order of a few months.

Acknowledgements. We would like to thank the anonymous referee for the helpful comments and suggestions. The authors acknowledge the use of data of the Kitt Peak telescope of the National Solar Observatory; NSO/Kitt Peak data used here are produced cooperatively by NSF/NOAO, NASA/GSFC, and NOAA/SEL. We also thank the Wilcox Solar Observatory for the use of the synoptic maps of photospheric magnetic field distribution. G.P. acknowledges support from ASI I/023/09/0.

\section{References}

Arnaud, M., \& Rothenflug, R. 1985, A\&AS, 60, 425

Baker, D., van Driel-Gesztelyi, L., Mandrini, C. H., Démoulin, P., \& Murray, M. J. 2009, ApJ, 705, 926

Bradshaw, S. J., Aulanier, G., \& Del Zanna, G. 2011, ApJ, 743, 66

Brooks, D. H., \& Warren, H. P. 2011, ApJ, 727, L13

Brooks, D. H., Ugarte-Urra, I., \& Warren, H. P. 2015, Nature Commun., 6, 5947

Brueckner, G. E., Howard, R. A., Koomen, M. J., et al. 1995, Sol. Phys., 162, 357

Cranmer, S. R. 2009, Liv. Rev. Sol. Phys., 6, 3

Cranmer, S. R., Gardner, L. D., \& Kohl, J. L. 2010, Sol. Phys., 263, 275

Culhane, J. L., Brooks, D. H., van Driel-Gesztelyi, L., et al. 2014, Sol. Phys., 289,3799

Delaboudinière, J.-P., Artzner, G. E., Brunaud, J., et al. 1995, Sol. Phys., 162, 291

Del Zanna, G. 2008, A\&A, 481, L49

Del Zanna, G., Aulanier, G., Klein, K.-L., \& Török, T. 2011, A\&A, 526, A137

Demoulin, P., Henoux, J. C., Priest, E. R., \& Mandrini, C. H. 1996, A\&A, 308, 643

Démoulin, P., Baker, D., Mandrini, C. H., \& van Driel-Gesztelyi, L. 2013, Sol. Phys., 283, 341

De Pontieu, B., \& McIntosh, S. W. 2010, ApJ, 722, 1013

Domingo, V., Fleck, B., \& Poland, A. I. 1995, Sol. Phys., 162, 1

Doschek, G. A., Warren, H. P., Mariska, J. T., et al. 2008, ApJ, 686, 1362

Frazin, R. A., Cranmer, S. R., \& Kohl, J. L. 2003, ApJ, 597, 1145

Gibson, S. E., Fludra, A., Bagenal, F., et al. 1999, J. Geophys. Res., 104, 9691

Green, L. M., López fuentes, M. C., Mandrini, C. H., et al. 2002, Sol. Phys., 208, 43
Guhathakurta, M., Fludra, A., Gibson, S. E., Biesecker, D., \& Fisher, R. 1999, J. Geophys. Res., 104, 9801

Hara, H., Watanabe, T., Matsuzaki, K., et al. 2008, PASJ, 60, 275

Harra, L. K., Sakao, T., Mandrini, C. H., et al. 2008, ApJ, 676, L147

Hyder, C. L., \& Lites, B. W. 1970, Sol. Phys., 14, 147

Ko, Y.-K., Raymond, J. C., Li, J., et al. 2002, ApJ, 578, 979

Ko, Y.-K., Raymond, J. C., Zurbuchen, T. H., et al. 2006, ApJ, 646, 1275

Kohl, J. L., Esser, R., Gardner, L. D., et al. 1995, Sol. Phys., 162, 313

Kohl, J. L., Noci, G., Antonucci, E., et al. 1997, Sol. Phys., 175, 613

Kohl, J. L., Noci, G., Cranmer, S. R., \& Raymond, J. C. 2006, A\&ARv, 13, 31

Kojima, M., Fujiki, K., Ohmi, T., et al. 1999, J. Geophys. Res., 104, 16993

Li, X., Habbal, S. R., Kohl, J. L., \& Noci, G. 1998, ApJ, 501, L133

Liewer, P. C., Neugebauer, M., \& Zurbuchen, T. 2004, Sol. Phys., 223, 209

Mandrini, C. H., Démoulin, P., van Driel-Gesztelyi, L., et al. 2004, Ap\&SS, 290, 319

Marsch, E., Tian, H., Sun, J., Curdt, W., \& Wiegelmann, T. 2008, ApJ, 685, 1262

Murray, M. J., Baker, D., van Driel-Gesztelyi, L., \& Sun, J. 2010, Sol. Phys., 261,253

Neugebauer, M., Liewer, P. C., Smith, E. J., Skoug, R. M., \& Zurbuchen, T. H. 2002, J. Geophys. Res. (Space Phys.), 107, 1488

Noci, G., \& Maccari, L. 1999, A\&A, 341, 275

Noci, G., Kohl, J. L., \& Withbroe, G. L. 1987, ApJ, 315, 706

Rust, D. M., Haggerty, D. K., Georgoulis, M. K., et al. 2008, ApJ, 687, 635

Sakao, T., Kano, R., Narukage, N., et al. 2007, Science, 318, 1585

Schrijver, C. J. 2001, ApJ, 547, 475

Schrijver, C. J., \& De Rosa, M. L. 2003, Sol. Phys., 212, 165

Schrijver, C. J., DeRosa, M. L., \& Title, A. M. 2010, ApJ, 719, 1083

Strachan, L., Baham, M., Miralles, M. P., \& Panasyuk, A. V. 2004, in SOHO 15 Coronal Heating, eds. R. W. Walsh, J. Ireland, D. Danesy, \& B. Fleck, ESA SP, 575,148

Tian, H., McIntosh, S. W., \& De Pontieu, B. 2011a, ApJ, 727, L37

Tian, H., McIntosh, S. W., De Pontieu, B., et al. 2011b, ApJ, 738, 18

van Driel-Gesztelyi, L., Culhane, J. L., Baker, D., et al. 2012, Sol. Phys., 281, 237

Vanninathan, K., Madjarska, M. S., Galsgaard, K., Huang, Z., \& Doyle, J. G. 2015, A\&A, 584, A38

von Steiger, R., Schwadron, N. A., Fisk, L. A., et al. 2000, J. Geophys. Res., 105, 27217

Wang, Y.-M. 2012, Space Sci. Rev., 172, 123

Warren, H. P., Mariska, J. T., Wilhelm, K., \& Lemaire, P. 1997, ApJ, 484, L91

Winebarger, A. R., DeLuca, E. E., \& Golub, L. 2001, ApJ, 553, L81

Withbroe, G. L., Kohl, J. L., Weiser, H., \& Munro, R. H. 1982, Space Sci. Rev., 33, 17

Woods, T. N., Tobiska, W. K., Rottman, G. J., \& Worden, J. R. 2000, J. Geophys. Res., 105, 27195

Yan, Y., Liu, Y., Akioka, M., \& Wei, F. 2001, Sol. Phys., 201, 337

Zangrilli, L., \& Poletto, G. 2012, A\&A, 545, A8

Zangrilli, L., Poletto, G., Nicolosi, P., Noci, G., \& Romoli, M. 2002, ApJ, 574, 477 